\title{
Taxa de permanência hospitalar de pacientes reinternados em hospital psiquiátrico
}

\author{
Length of stay of patients hospitalized at a psychiatric hospital \\ Vanessa Machadoํ, Manoel Antônio dos Santos
}

\section{RESUMO}

Objetivo: Analisar a relação entre características sociodemográicas e taxa de permanência hospitalar, entendida como a razão entre a quantidade total de dias internado e o número total de internações, em 114 pacientes reinternantes em hospital psiquiátrico estadual. Métodos: Os dados demográficos referentes a sexo, idade, estado civil, nível de instrução e cor foram levantados nos prontuários médicos dos pacientes. Já dados sobre os aspectos sociais referentes a situação ocupacional, recebimento de benefício, residência, acompanhantes, visitas e telefonemas durante a internação foram colhidos por meio da aplicação de um questionário aos profissionais do hospital. Resultados: A análise estatística mostrou que os sujeitos que apresentaram maior taxa de permanência hospitalar foram do sexo feminino, entre 40 e 49 anos, viúvos ou divorciados, negros, que residiam com outras pessoas e que

\section{Palavras-chave}

Readmissão, taxa de permanência, apoio social, saúde mental.

\section{Keywords}

Readmission, length of stay, social support, mental health. não recebiam visitas durante o período em que se encontravam internados. Conclusão: Esses achados apontam para a existência de relação entre características sociodemográficas e taxa de permanência, sugerindo a importância da rede de apoio social na reabilitação do pacientes com história de internações recorrentes.

\section{ABSTRACT}

Objective: To analyze the relationship between socio-demographic characteristics and the permanence rate, i.e., the ratio between the total number of days in hospital and the total number of admissions, among 114 patients readmitted to a public psychiatric hospital. Methods: Demographic data related to gender, age, marital status, instruction level and color were surveyed in the patients' medical records. Hospital professionals received a questionnaire, asking for data on social aspects related to the occupational situation, reception of financial aid, living with other persons, company when arriving for hospitalization, receiving visits and phone calls during the hospitalization. Results: Statistical analysis showed that female individuals aged 40-49 years, divorced or widowed, black, living with other people and who did not receive any visits while hospitalized stayed longer in hospital. Conclusion: These findings suggest that socio-demographic characteristics are associated with length of stay, indicating the relevance of the social support network for the recovery of patients with a history of recurring admissions. 


\section{INTRODUÇÃO}

Ao se considerarem as mudanças na abordagem da saúde mental, que passou a priorizar o modelo de atenção comunitária em oposição ao modelo centrado no hospital, o tempo de permanência hospitalar, associado a outros indicadores, tem sido acatado na avaliação da qualidade da assistência em saúde mental. Na literatura norte-americana, os pacientes com histórico de internações por longo tempo de permanência são denominados heavy users ${ }^{1,2}$. As características sociodemográficas e clínicas desses indivíduos têm sido foco de interesse crescente nos últimos anos.

Estudos realizados nos Estados Unidos em unidades psiquiátricas de hospitais gerais identificaram um tempo médio de internação de $23^{3}$ a $21^{4}$ dias. Pesquisa realizada por Caton identificou que o tempo de internação de 119 pacientes esquizofrênicos em seis instituições urbanas norte-americanas variou de 21 dias em unidades para pacientes agudos até 70 dias em unidades estaduais de longa permanência e que a duração da hospitalização não tinha qualquer relação com a internação posterior, adesão ao tratamento clínico ou com o funcionamento social na comunidade ${ }^{5}$.

No cenário brasileiro, investigações ${ }^{6}$ realizadas entre dezembro de 1986 e novembro de 1988 identificaram que o tempo médio de permanência em uma unidade psiquiátrica de um hospital geral universitário era de 19,4 dias, período relativamente menor do que o observado em hospital psiquiátrico. Estudos realizados na década de 1990 demonstraram que a média nacional do tempo de permanência de internação psiquiátrica era de 18,6 dias, e nos hospitais psiquiátricos conveniados ao Sistema Único de Saúde (SUS), essa média era maior (24 dias) do que as internações psiquiátricas em hospitais gerais universitários (14 dias) ${ }^{7}$.

Outras investigações identificaram ainda um tempo médio de internação em hospitais psiquiátricos conveniados ao SUS de 31,85 dias $^{8}$ e de 30 dias $^{9}$. Em censo nacional realizado por Botega e Schechtman ${ }^{10}$, identificou-se que o tempo médio de internação em hospitais psiquiátricos era de cerca de 20 dias, significativamente superior ao tempo médio de internação em unidades psiquiátricas de hospitais gerais integrados ao SUS, que era de cinco a sete dias. De acordo com os resultados desses estudos, portanto, o tempo de permanência hospitalar parece ser maior quando a internação é realizada em hospital psiquiátrico.

Por outro lado, investigação ${ }^{11}$ realizada no município de Ribeirão Preto concluiu que na unidade de emergência do hospital universitário prevaleceram internações que duraram menos de dois dias, no hospital psiquiátrico estadual predominaram as inferiores a 20 dias e no hospital geral universitário prevaleceram internações por mais de 20 dias. Essa diferença, segundo o autor, possivelmente pode ser justificada pela gravidade e comorbidade dos casos atendidos pelo hospital universitário.
Reconhecido indicador de qualidade da assistência à saúde mental, o fenômeno da reinternação psiquiátrica, expresso por meio da taxa de reinternação, tem sido denominado de "porta giratória", ou "revolving door", por alguns autores $^{12-14}$. Nos estudos norte-americanos, o paciente reinternante tem sido designado como "frequent users". Esse fenômeno explicita que, embora se tenha reduzido o tempo das internações, que em outros tempos era de longa permanência, a institucionalização dos pacientes acontece por meio de internações relativamente breves, que passam a transitar por diferentes hospitais e, assim, caracterizar o uso frequente da internação ${ }^{15,16}$.

A reinternação é um fenômeno de múltiplas determinações, associado especialmente a diagnóstico ${ }^{1,17-19}$, características sociodemográficas ${ }^{1,18,20}$, adesão ao tratamento ambulatorial $^{21,22}$ e qualidade e modalidade do tratamento ambulatorial oferecido pela rede ${ }^{23,24}$. Em geral, a reinternação é definida por um determinado número de internações em um período limitado, como duas ou mais internações em um ano 18,19,25,26, três ou mais internações em um ano ${ }^{27}$, três ou mais internações em um período de um ano e meio ${ }^{28}$. Neste estudo, foram considerados pacientes reinternantes aqueles que, ao longo de sua vida, apresentaram mais de uma internação no hospital psiquiátrico no qual se desenvolveu a investigação.

Tendo em vista a importância do tema no atual cenário da saúde mental, assim como a ausência de estudos que investiguem a associação entre taxa de permanência e aspectos sociodemográficos em pacientes reinternantes, delineou-se o presente estudo. Assim, este estudo teve por objetivo identificar os fatores sociodemográficos associados à taxa de permanência hospitalar, entendida como a razão entre a quantidade total de dias internado e o número total de internações, entre pacientes reinternantes em um hospital psiquiátrico.

\section{METODOLOGIA}

\section{Local do estudo}

O estudo foi desenvolvido em um hospital psiquiátrico da Secretaria da Saúde do Estado de São Paulo, referência para o atendimento da população de 26 municípios, que totaliza 1.284.340 habitantes (IBGE, 2009). O hospital conta com dois tipos de atendimento: um destinado aos pacientes de longa permanência hospitalar, remanescentes da antiga política de institucionalização, e outro setor que oferece assistência integral, por meio de internações chamadas breves, para pacientes em quadro agudo e dependentes químicos. No período de coleta de dados da presente pesquisa, o setor de internação breve era subdividido da seguinte forma: setor de internação para pacientes do sexo feminino (chamado "agudos feminino", com 30 leitos), para pacientes do sexo masculino (chamado "agudos masculino", com 30 leitos) e para pacientes do sexo masculino com dependência quími- 
ca (chamado "dependentes químicos", com 20 leitos). Todos os setores contam com duas equipes multidisciplinares, nos períodos de manhã e da tarde, bem como profissionais médicos e assistência de enfermagem em período integral.

\section{Sujeitos}

Definiram-se como critérios de inclusão dos sujeitos na amostra: pacientes que haviam apresentado reinternação nos setores de internação breve no hospital psiquiátrico de interesse da pesquisa, no período de julho a dezembro de 2006; residentes no município de Ribeirão Preto, interior do estado de São Paulo, onde a referida instituição está localizada. Estabeleceram-se os seguintes critérios de exclusão: pacientes internados pela primeira vez no hospital; residentes em outros municípios que não o de interesse.

Para a composição da amostra, foi realizado um levantamento por meio de uma pesquisa preliminar na rede de sistema de arquivo médico desse hospital. Em um primeiro momento, foram levantadas todas as admissões de pacientes na instituição hospitalar no período de julho a dezembro de 2006, totalizando 547 admissões. Em seguida, foram aplicados os critérios de inclusão/exclusão, que resultaram em um total de 182 internações realizadas por 141 pacientes. Portanto, para a composição da amostra $(N=141)$ foi considerada a totalidade dos pacientes reinternados no período definido pelo estudo nos três setores de internação breve do hospital psiquiátrico, residentes no município de Ribeirão Preto.

\section{Coleta de dados}

Como instrumento, foi elaborado e previamente testado um formulário para caracterização sociodemográfica, que se mostrou adequado para a coleta dos dados de interesse. O formulário foi composto de duas partes: informações coletadas a partir de dados secundários e informações coletadas a partir de uma entrevista estruturada aplicada aos profissionais do hospital. Os dados secundários foram extraídos de informações constantes dos prontuários médicos de cada um dos sujeitos (data de admissão e alta de todas as internações, sexo, idade, estado civil, nível de instrução e cor). Os dados que diziam respeito aos aspectos sociais foram respondidos por profissionais técnicos da instituição, escolhidos entre aqueles que estivessem mais habilitados a responder pelos cuidados prestados aos sujeitos durante suas internações, aos quais foi aplicada uma entrevista estruturada que contemplou as seguintes questões: Qual a atual situação ocupacional do paciente? (ativo, inativo); O paciente recebe algum tipo de benefício? (sim, não); O paciente reside com outras pessoas? (sim, não); O paciente geralmente chega acompanhado nas internações? (sim, não); O paciente geralmente recebe visita enquanto está internado? (sim, não); O paciente geralmente recebe telefonema enquanto está internado? (sim, não).

Este estudo foi aprovado pelo Comitê de Ética em Pesquisa do Centro de Saúde Escola da Faculdade de Medicina de Ribeirão Preto - Universidade de São Paulo (protocolo n 229).

\section{Análise de dados}

Ao considerar que o tempo de observação de cada sujeito era variado, uma vez que as datas das primeiras internações diferiam entre eles, optou-se por trabalhar com a taxa de permanência hospitalar, que representa a razão entre a quantidade total de dias internado e o número total de internações. Sendo assim, a taxa de permanência é uma variável discreta, que indica a proporção de tempo que cada sujeito permaneceu no sistema.

Por haver apenas uma variável dependente (taxa de permanência), foi realizada uma análise univariada. Tendo em vista a grande dispersão da variável dependente, ela foi ajustada por meio de um modelo de regressão binomial negativa, em função das variáveis independentes: idade, estado civil, sexo, entre outras. Como a variável dependente é discreta e representa uma razão, é plausível utilizar esse modelo para análise dos dados, considerando, ainda, que se pode presumir a superdispersão dos dados. Além disso, foi ajustado um modelo para cada uma das variáveis independentes, e a qualidade do ajuste, que foi avaliada via scaled deviance, não se afastou significativamente do valor 1. Considerando um nível de significância de 5\%, o ajuste univariado do modelo apontou como sendo significantes as variáveis que retornaram $p$-valor $<0,05$.

\section{RESULTADOS}

A título de descrição da amostra, a taxa de permanência hospitalar foi de 33,5 dias para os pacientes internados nos setores agudos feminino e agudos masculino e de 14,5 dias para os pacientes internados no setor de dependentes químicos. Os pacientes internados nos setores agudos feminino e agudos masculino apresentaram um intervalo médio entre as internações de 323,5 dias e uma média de 7,9 internações; os pacientes internados no setor dependentes químicos apresentaram o intervalo médio de internações de 556,8 dias e média de 4,8 internações.

A análise estatística apontou diferenças estaticamente significantes no que se refere às variáveis independentes: sexo, faixa etária, estado civil e cor. Os sujeitos do sexo feminino apresentaram uma taxa de permanência de 36 dias, enquanto os sujeitos do sexo masculino, em média, permaneceram no hospital por 17,3 dias. No que diz respeito à faixa etária, os sujeitos na faixa etária de 40 a 49 anos apresentaram a maior taxa de permanência (36,4 dias), seguidos dos sujeitos com idade igual ou maior de 50 anos (23,5 dias), na faixa etária de 30 a 39 anos (18,4 dias) e 29 anos ou menos (17,7 dias). Quanto ao estado civil, os sujeitos viúvos ou divorciados, em média, permaneceram internados 25,1 dias, enquanto os solteiros permaneceram 20,2 dias e os casados, 17,7 dias. No que concerne à cor, os sujeitos de cor negra permaneceram, em média, 71,8 dias internados, enquanto os de cor parda permaneceram 20,3 dias e os de cor branca, 20,1 dias (Tabela 1). 
No que diz respeito aos aspectos sociais, a análise estatística mostrou diferenças estaticamente significantes no que concerne ao paciente residir com outras pessoas $(p=0,009)$, bem como receber visitas enquanto se encontra internado ( $p<0,0001)$. Os sujeitos que residem com outras pessoas apresentaram, em média, um tempo de permanência hospitalar de 31,07 dias, enquanto aqueles que não residem com outras pessoas apresentaram uma taxa de permanência de 18,3 dias. Os sujeitos que recebem visitas de um membro da rede social enquanto estão internados apresentaram a taxa de permanência de 22,3 dias, enquanto os sujeitos que não recebem essas visitas apresentaram uma taxa de 26,4 dias (Tabela 2).

Tabela 1. Distribuição da taxa de permanência hospitalar em função do sexo, faixa etária, estado civil, nível de instrução e cor

\begin{tabular}{|c|c|c|c|c|c|c|c|}
\hline Variáveis & $\begin{array}{l}\text { Taxa de permanência } \\
\text { (dias) }\end{array}$ & Desvio-padrão & $\begin{array}{l}\text { Coeficiente de } \\
\text { variação (\%) }\end{array}$ & Mínimo & Mediana & Máximo & P-valor \\
\hline \multicolumn{8}{|l|}{ Sexo } \\
\hline Feminino & 36 & 97,32 & 270,35 & 2 & 17,81 & 724 & \multirow{2}{*}{$<0,0001^{*}$} \\
\hline Masculino & 17,35 & 13,50 & 77,79 & 4 & 13,43 & 106 & \\
\hline \multicolumn{8}{|l|}{ Faixa etária (anos) } \\
\hline$\leq 29$ & 17,72 & 10,29 & 58,07 & 2 & 14,08 & 46 & \multirow{4}{*}{$<0,0001^{*}$} \\
\hline $30-39$ & 18,48 & 21,54 & 116,55 & 5 & 12,39 & 128 & \\
\hline $40-49$ & 36,43 & 112,13 & 307,75 & 4 & 16,38 & 724 & \\
\hline$\geq 50$ & 23,58 & 19,99 & 84,75 & 5 & 19,00 & 106 & \\
\hline \multicolumn{8}{|l|}{ Estado civil } \\
\hline Casado & 17,77 & 10,99 & 61,84 & 2 & 15,58 & 49 & \multirow{3}{*}{$0,0003^{*}$} \\
\hline Solteiro & 20,24 & 16,94 & 83,72 & 5 & 15,27 & 128 & \\
\hline Viúvo/divorciado & 25,16 & 24,29 & 96,53 & 7 & 19 & 106 & \\
\hline \multicolumn{8}{|l|}{ Nível de instrução } \\
\hline Sem estudo & 20,47 & 17,66 & 86,25 & 12 & 13,83 & 64 & \multirow{7}{*}{0,1504} \\
\hline Fund. incomp. & 30,86 & 92,23 & 298,90 & 5 & 19 & 724 & \\
\hline Fund. comp. & 19,72 & 9,97 & 50,56 & 5 & 18,25 & 35 & \\
\hline Médio incompl. & 20,40 & 14,36 & 70,40 & 8 & 16,54 & 46 & \\
\hline Médio compl. & 13,96 & 10,30 & 73,75 & 2 & 11 & 39 & \\
\hline Superior incomp. & 18,50 & 19,07 & 103,08 & 7 & 9,50 & 57 & \\
\hline Superior compl. & 17,29 & 18,79 & 108,70 & 4 & 17,29 & 31 & \\
\hline \multicolumn{8}{|l|}{ Cor } \\
\hline Branca & 20,12 & 15,69 & 77,96 & 2 & 15,38 & 128 & \multirow{3}{*}{$0,04^{*}$} \\
\hline Negra & 71,82 & 188,45 & 262,38 & 7 & 18,10 & 724 & \\
\hline Parda & 20,30 & 22,33 & 110,03 & 7 & 14,53 & 106 & \\
\hline
\end{tabular}

${ }^{*} p<0,05$

Tabela 2. Distribuição da taxa de permanência hospitalar em função da situação ocupacional, do recebimento de benefício, de residir com outras pessoas, de chegar acompanhado nas internações, de receber visitas e telefonemas enquanto está internado

\begin{tabular}{|c|c|c|c|c|c|c|c|}
\hline Variável & $\begin{array}{l}\text { Taxa de permanência } \\
\text { (dias) }\end{array}$ & Desvio-padrão & $\begin{array}{l}\text { Coeficiente de } \\
\text { variação }(\%)\end{array}$ & Mínimo & Mediana & Máximo & P-valor \\
\hline \multicolumn{8}{|c|}{ Situação ocupacional } \\
\hline Econ. ativo & 17,84 & 11,39 & 63,83 & 2 & 14,5 & 39 & \multirow{2}{*}{0,7869} \\
\hline Econ. inativo & 33,15 & 83,75 & 252,59 & 5 & 19,83 & 724 & \\
\hline \multicolumn{8}{|c|}{ Recebimento benefício } \\
\hline $\operatorname{Sim}$ & 41,23 & 109,28 & 265,03 & 5 & 17,5 & 724 & \multirow{2}{*}{0,0543} \\
\hline Não & 20,43 & 11,27 & 55,15 & 2 & 19,33 & 57 & \\
\hline \multicolumn{8}{|c|}{ Reside c/ outra pessoa } \\
\hline Sim & 31,07 & 78,4 & 252,33 & 2 & 19 & 724 & \multirow{2}{*}{$0,0089^{*}$} \\
\hline Não & 18,30 & 9,41 & 51,44 & 5 & 17 & 33 & \\
\hline \multicolumn{8}{|c|}{ Chega acompanhado nas internações } \\
\hline Sim & 22,97 & 17,73 & 77,19 & 7 & 18,10 & 106 & \multirow{2}{*}{0,1032} \\
\hline Não & 22,08 & 20,46 & 92,67 & 2 & 19 & 128 & \\
\hline \multicolumn{8}{|c|}{ Recebe visita enquanto está internado } \\
\hline Sim & 22,34 & 17,88 & 80,05 & 5 & 17,81 & 128 & \multirow{2}{*}{$<0,0001^{*}$} \\
\hline Não & 26,46 & 8,74 & 33,03 & 17 & 27,29 & 35 & \\
\hline \multicolumn{8}{|c|}{ Recebe telefonema enquanto está internado } \\
\hline $\operatorname{Sim}$ & 23,08 & 19,25 & 83,40 & 5 & 17,33 & 128 & \multirow{2}{*}{0,6264} \\
\hline Não & 20,23 & 7,80 & 38,57 & 8 & 19,83 & 33 & \\
\hline
\end{tabular}


Tabela 3. Distribuição da taxa de permanência hospitalar em função da forma de demonstração de preocupação/cuidado com o paciente

\begin{tabular}{|c|c|c|c|c|c|c|c|}
\hline Forma de demonstração de preocupação/cuidado & $\begin{array}{l}\text { Taxa de } \\
\text { permanência } \\
\text { (dias) }\end{array}$ & Desvio-padrão & $\begin{array}{l}\text { Coeficiente de } \\
\text { variaçąa }(\%)\end{array}$ & Mínimo & Mediana & Máximo & P-valor \\
\hline \multicolumn{8}{|l|}{ Visita o paciente enquanto está internado } \\
\hline $\operatorname{Sim}$ & 22,34 & 17,88 & 80,05 & 5 & 17,81 & 128 & \multirow{2}{*}{$<0,0001^{*}$} \\
\hline Não & 26,46 & 8,74 & 33,03 & 17 & 27,29 & 35 & \\
\hline \multicolumn{8}{|c|}{ Telefonema para o paciente enquanto está internado } \\
\hline Sim & 23,08 & 19,25 & 83,40 & 5 & 17,33 & 128 & \multirow{2}{*}{0,6264} \\
\hline Não & 20,23 & 7,80 & 38,57 & 8 & 19,83 & 33 & \\
\hline \multicolumn{8}{|l|}{ Demonstra afetividade para com o paciente } \\
\hline $\operatorname{Sim}$ & 23,97 & 21,03 & 87,72 & 7 & 19 & 128 & \multirow{2}{*}{0,4858} \\
\hline Não & 20,74 & 12,24 & 59,00 & 5 & 17,67 & 57 & \\
\hline \multicolumn{8}{|c|}{ Demonstra satisfação no momento da alta e/ou da alta licença do paciente } \\
\hline $\operatorname{Sim}$ & 24,22 & 21,04 & 86,88 & 7 & 17,56 & 128 & \multirow{2}{*}{0,3193} \\
\hline Não & 20,32 & 11,69 & 57,52 & 5 & 19 & 57 & \\
\hline \multicolumn{8}{|l|}{ Acompanha nas consultas médicas } \\
\hline Sim & 24,24 & 20,82 & 85,91 & 7 & 19 & 128 & \multirow{2}{*}{0,2357} \\
\hline Não & 19,71 & 10,08 & 51,14 & 5 & 17,50 & 43 & \\
\hline \multicolumn{8}{|l|}{ Ministra as medicações } \\
\hline Sim & 27,01 & 21,76 & 80,58 & 7 & 20,22 & 128 & \multirow{2}{*}{0,1732} \\
\hline Não & 18,15 & 10,87 & 59,88 & 5 & 16,98 & 57 & \\
\hline \multicolumn{8}{|c|}{ Cuida das tarefas da casa do paciente e/ou dos seus filhos } \\
\hline $\operatorname{Sim}$ & 23,01 & 21,52 & 93,52 & 7 & 16,50 & 128 & \multirow{2}{*}{$0,006^{*}$} \\
\hline Não & 21,91 & 11,37 & 51,90 & 5 & 19,63 & 57 & \\
\hline
\end{tabular}

\section{DISCUSSÃO}

Em termos de estimativa populacional para a população do município de Ribeirão Preto reinternante no hospital investigado, verificou-se que algumas variáveis demonstraram exercer efeito sobre a taxa de permanência hospitalar. Os dados permitem estimar que, considerando os limites dessa população, os sujeitos do sexo feminino apresentam maior taxa de permanência hospitalar. Esse dado não pode ser explicado por meio da associação com a variável diagnóstico, como se poderia supor em exame preliminar, já que, em análise paralela, essa variável não trouxe efeitos na taxa de permanência. Por outro lado, pode ser associado ao setor de internação, considerando que, na época do presente estudo, homens e mulheres eram internados em setores separados, com equipes e gerenciamentos também distintos. Assim, esse achado permite inferir possíveis diferenças sociais e clínicas inerentes ao gênero, mas também se deve considerar a influência de diferenças na condução e manejo dos casos pelas diferentes equipes de saúde mental.

A equipe, ao discutir as possibilidades de alta do paciente, além de levar em consideração o estado clínico, também avalia o suporte social com que ele conta para apoiá-lo naquele determinado momento, assim como as características do serviço ambulatorial responsável por seu acompanhamento extra-hospitalar. Essa situação indica que são vários os aspectos, não só clínicos, presentes na avaliação de uma possível alta, o que deixa margem para diferentes avaliações e possibilidades de encaminhamentos dos casos, conforme as discussões e negociações estabelecidas pelos profissionais de saúde.

Outro aspecto importante a ser problematizado é a pressão da rede de assistência por mais vagas, especialmente nos últimos anos, o que pode favorecer que se acelere o processo de alta. Segundo levantamento no serviço de arquivo médico e estatístico do hospital psiquiátrico investigado, a taxa de ocupação relacionada a internações de pacientes do sexo masculino foi sempre superior às internações de pacientes do sexo feminino; só nos últimos anos houve uma equiparação. Esse aspecto pode indicar que as possíveis pressões da rede, que demanda vagas para internação, estejam interferindo em uma alta mais breve no setor de internação para pacientes do sexo masculino, implicando uma acentuada diferença entre o tempo de permanência desse setor e do setor de internação para mulheres.

No que se refere à faixa etária, a partir da análise estatística dos dados, pode-se estimar que sujeitos na meia-idade (entre 40 e 49 anos) apresentam maior taxa de permanência, o que permite inferir que os mais jovens apresentam uma remissão mais rápida dos sintomas, que se mostra mais dificultada em sujeitos de maior idade, tendo em vista a evolução crônica do quadro. Estudos desenvolvidos com pacien- 
tes psiquiátricos apontam que idade igual ou superior a 60 anos contribui para o aumento no tempo de permanência ${ }^{29}$, contudo não se trata de uma população exclusivamente de pacientes reinternantes, o que pode sugerir que relativamente a essa população o tempo de permanência é aumentado mais precocemente.

Os sujeitos que tiveram o vínculo conjugal rompido, seja por separação (divórcio) ou viuvez, apresentam maior taxa de permanência hospitalar em comparação com pacientes solteiros e casados. A partir desse dado, pode-se hipotetizar que o cônjuge desempenha um papel importante na não institucionalização prolongada do paciente. De forma semeIhante, os sujeitos da cor negra apresentaram uma taxa de permanência hospitalar muito maior do que os demais. Esse achado vai ao encontro da histórica exclusão social, estigmatização e consequente marginalização sofrida por esse segmento da população.

Quanto a residir com outra pessoa, chama a atenção o dado que mostra que os sujeitos que residem com outros apresentam maior taxa de permanência hospitalar. Pode-se pensar que o fato de ter família ou residir com outra pessoa não assegura a qualidade dos relacionamentos interpessoais, tal como se pode encontrar nos vínculos permeados por demonstrações de preocupação ou cuidado. Corroborando tal interpretação, por meio dos dados analisados, estima-se que os sujeitos que recebem visitas enquanto estão internados apresentam menor taxa de permanência hospitalar. Esse dado sugere que a manutenção do contato com pessoas de sua rede social exerce contribuição positiva à recuperação do paciente, corroborando outros estudos ${ }^{30}$.

Este estudo apresenta algumas limitações. A delimitação da população do estudo (reinternantes em um único hospital, público, que atende a uma área específica do estado de São Paulo) não permite a generalização dos resultados para outros contextos. Ainda, considerando que foram entrevistados diferentes profissionais, não houve uniformidade das respostas, uma vez que elas estavam sujeitas à compreensão subjetiva dos entrevistados.

\section{CONCLUSÃO}

A partir da análise estatística, conclui-se que os pacientes psiquiátricos reinternantes que apresentam maior taxa de permanência hospitalar são do sexo feminino, com idade entre 40 e 49 anos, viúvos ou divorciados, negros, que residem com outras pessoas, não recebem visitas durante o período em que se encontram internados. Esses achados sugerem que características sociodemográficas interferem na taxa de permanência hospitalar, apontando para a concepção da importância dos aspectos sociais na reabilitação psicossocial e na manutenção da estabilidade do quadro psiquiátrico.
Assim, deve-se considerar que aspectos não meramente clínicos interferem na decisão sobre a alta hospitalar, e o quadro clínico não é balizador suficiente para consubstanciar tal avaliação. Geralmente, entram em questão a apreciação da rede social e familiar de apoio e as condições de atendimento do serviço ambulatorial de contrarreferência. Ainda, além das questões de cunho social e a disponibilidade e qualidade da rede de serviços de saúde mental, existem as questões institucionais, como é o caso das internações por determinação judicial, que têm se tornado cada vez mais frequentes, sendo responsáveis por consideráveis aumentos no tempo de permanência na internação.

Em um momento no qual o processo de desinstitucionalização encontra-se em curso, os indicadores taxa de permanência e taxa de reinternações psiquiátricas oferecem recursos para a avaliação do funcionamento da rede de serviços à saúde mental, nas suas dimensões hospitalar, extra-hospitalar e social, que não podem ser analisadas de forma isolada e fragmentada.

\section{AGRADECIMENTO}

Agradecemos pelo apoio recebido da Fundação de Amparo à Pesquisa do Estado de São Paulo (FAPESP) ao projeto de pesquisa do Programa de Pesquisa em Políticas Públicas intitulado "Avaliação de internações psiquiátricas na região de Ribeirão Preto - SP entre 1998-2004 e suas relações com a rede extra-hospitalar de saúde mental", processo no 06/51736-6, que gerou este artigo.

\section{REFERÊNCIAS}

1. Lay B, Lauber C, Rãssler W. Prediction of in-patient use in first-admitted patients with psychosis. Eur Psychiatry. 2006;21(6):401-9.

2. Montgomery P, Kirkpatrick H. Understanding those who seek frequent psychiatric hospitalizations. Arch Psychiatr Nurs. 2002;16(1):16-24.

3. Lieberman PB, McPhetres EB, Elliott B, Egerter E, Wiitala S. Dimensions and predictors of change during brief psychiatric hospitalization. Gen Hosp Psychiatry. 1993;15(5):316-24.

4. Hibbard T, Trimboli F. Correlates of successful short-term psychiatric hospitalization. Hosp Community Psychiatr. 1982;33:829-33.

5. Caton CLM. Effect of length of inpatient treatment for chronic schizophrenia. Am J Psychiatry. 1982;139(7):856-61.

6. Dalgalarrondo P. Duração da internação em uma unidade psiquiátrica de hospital geral: a experiência do Hospital das Clínicas da UNICAMP. J Bras Psiquiatr. 1993;42(1):15-7.

7. Ribeiro MS, Siano AK, Procópio NA, Santos KHO, Alves MJM. Psychiatry in general hospital versus psychiatric hospital: an initial study of evaluation of the assistance performed by the psychiatric residency of the HU-UF JF. HU Revista. 2000;25(3):167-79.

8. Souza JC, Souza N, Caetano D. Tempo médio de hospitalização em um hospital psiquiátrico de Campo Grande, Mato Grosso do Sul. J Bras Psiquiatr. 2005;54(1):13-8.

9. Brenner MK, Cardoso VM, Oliveira RL, Fensterseifer, GP, Barraz, ACG, Boeira LB, et al. Mudanças na clientela de internados em hospital psiquiátrico. J Bras Psiquiatr. 1999;48(9):415-20.

10. Botega NJ, Schechtman A. Censo nacional de unidades de psiquiatria em hospitais gerais. I Situação atual e tendências. Revista ABP-APAL. 1997;19(3):79-86. 
11. Barros RE. Caracterização clínica-demográfica das internações psiquiátricas na região de Ribeirão Preto-SP entre 1998 e 2004 [dissertação]. Ribeirão Preto: Faculdade de Medicina de Ribeirão Preto, Universidade de São Paulo. Ribeirão Preto; 2008.

12. Geller MD. The "revolving door": a trap or a life style? Hosp Community Psychiatr. 1982;33(5):388-9.

13. Rosenfeld AH. Closing the revolving door through family therapy. Hosp Community Psychiatr. 1982;33(6):893-4.

14. Bandeira M. Desinstitucionalização ou transinstitucionalização: lições de alguns países. J Bras Psiquiatr. 1991;40(7):335-60.

15. Dalgalarrondo P. Enfermarias de psiquiatria. In: Botega NJ, organizador. Serviços de saúde mental no hospital geral. Campinas: Papiros; 1995

16. Mello R, Furegato ARF. Internações psiquiátricas no Rio de Janeiro de 1996 a 2005. R Enferm UERJ. 2007;15(2):176-82.

17. Amaral MA. Saúde mental na rede básica: modelo assistencial. Rev Saude Publica 1997;31(3):288-95.

18. Parente CJS, Mendes LPF, Souza CNS, Silva DKM, Silva JC, Parente ACBV, et al. 0 fenômeno de revolving door em hospitais psiquiátricos de uma capital do Nordeste brasileiro. REME Rev Min Enferm. 2007;11(4):381-6.

19. Saarento 0, Christiansen L, Gostas G, Kakko H, Lonnerberg O, Muus S, et al. The Nordic comparative study on sectorised pscychiatry: repeated emergency admissions to inpatient care during a 1-year follow-up. Eur Psychiatry. 1998;13(8):385-91.

20. Geller JL, Fisher WH, McDermeit M, Brown JM. The effects of public managed care on patterns of intensive use of inpatient psychiatric services. Psychiatr Serv. 1998:49(3):327-32.
21. Swartz MS, Swanson JW, Hiday VA, Wagner HR, Burns BJ, Borum R. A randomized controlled trial of outpatient commitment in North Carolina. Psychiatr Serv. 2001;52(3):325-9.

22. Suzuki Y, Yasumura S, Fukao A, Otani K. Associated factors of rehospitalization among schizophrenic patients. Psychiatry Clin Neurosci. 2003;57(6):555-61.

23. Chinman MJ, Weingarten R, Stayner D, Davidson L. Chronicity reconsidered: improving person-environment fit through a consumer-run service. Community Ment Health J. 2001;37(3):215-29.

24. Essock SM, Frisman LK, Kontos NJ. Cost-effectiveness of assertive community treatment teams. Am J Orthopsychiatry. 1998;68(2):179-90.

25. Sytema S, Philip B, Tansella M. Does community care decrease length of stay and risk of rehospitalization in new patients with schizophrenia disorders? A comparative case register study in Groningen, the Netherlands; Victoria, Australia; and South-Verona, Italy. Shizophr Bull. 2002;28(2):273-81.

26. Husted J, Jorgens A. Best practices: population density as a factor in the rehospitalization of persons with serious and persistent mental illness. Psychiatr Serv. 2000;51(5):603-5.

27. Korkeila JA, Lehtinen V, Tuori T, Helenius H. Patterns of psychiatric hospital service use in Finland: a national register study of hospital discharges in the early 1990s. Soc Psychiatry Psychiatr Epidemiol. 1998;33(5):218-23.

28. Kumar S, Robinson E, Kumar Sinha V. What leads to frequent re-hospitalisation when community care is not well developed? Soc Psychiatry Psychiatr Epidemiol. 2002:37(9):435-40.

29. Dalgalarrondo P, Botega NJ, Banzato CEM. Pacientes que se beneficiam de internação psiquiátrica em hospital geral. Rev Saude Publica. 2010;37(5)

30. Mezzich JE, Coffman GA. Factors influencing length of hospital stay. Hosp Community Psychiatry. 1985;36:1262-70 